\title{
Epidemiological Investigation of the First 135 COVID-19 Cases in Brunei: Implications for Surveillance, Control, and Travel Restrictions
}

\author{
Justin Wong, ${ }^{1} \dagger$ Liling Chaw,${ }^{2 *}+$ Wee Chian Koh, ${ }^{3}$ Mohammad Fathi Alikhan, ${ }^{1}$ Sirajul Adli Jamaludin, ${ }^{4}$ Wan Wen Patricia Poh, ${ }^{5}$ and \\ Lin Naing ${ }^{2}$ \\ ${ }^{1}$ Disease Control Division, Ministry of Health, Bandar Seri Begawan, Brunei Darussalam; ${ }^{2}$ PAPRSB Institute of Health Sciences, Universiti Brunei \\ Darussalam, Gadong, Brunei Darussalam; ${ }^{3}$ Centre for Strategic and Policy Studies, Bandar Seri Begawan, Brunei Darussalam; ${ }^{4}$ Environmental \\ Health Division, Ministry of Health, Bandar Seri Begawan, Brunei Darussalam; ${ }^{5}$ Department of Dental Services, Ministry of Health, Bandar Seri
} Begawan, Brunei Darussalam

\begin{abstract}
Studies on the early introduction of SARS-CoV-2 in a naive population have important epidemic control implications. We report findings from the epidemiological investigation of the initial 135 COVID-19 cases in Brunei and describe the impact of control measures and travel restrictions. Epidemiological and clinical information was obtained for all confirmed COVID-19 cases, whose symptom onset was from March 9 to April 5, 2020. The basic reproduction number (R0), incubation period, and serial interval (SI) were calculated. Time-varying $R$ was estimated to assess the effectiveness of control measures. Of the 135 cases detected, $53(39.3 \%)$ were imported. The median age was $36($ range $=0.5-72)$ years. Forty-one (30.4\%) and $13(9.6 \%)$ were presymptomatic and asymptomatic cases, respectively. The median incubation period was 5 days (interquartile range $[\mathrm{IQR}]=5$, range $=1-11)$, and the mean SI was 5.4 days $(\mathrm{SD}=4.5 ; 95 \% \mathrm{Cl}: 4.3,6.5)$. The reproduction number was between 3.9 and 6.0, and the doubling time was 1.3 days. The time-varying reproduction number $(\mathrm{Rt})$ was below one ( $\mathrm{Rt}=0.91 ; 95 \%$ credible interval: $0.62,1.32)$ by the 13 th day of the epidemic. Epidemic control was achieved through a combination of public health measures, with emphasis on a test-isolate-trace approach supplemented by travel restrictions and moderate physical distancing measures but no actual lockdown. Regular and ongoing testing of high-risk groups to supplement the existing surveillance program and a phased easing of physical distancing measures has helped maintain suppression of the COVID-19 outbreak in Brunei, as evidenced by the identification of only six additional cases from April 5 to August 5, 2020.
\end{abstract}

\section{INTRODUCTION}

The global spread of COVID-19 and the lack of an effective vaccine or therapeutic options pose challenges for disease control ${ }^{1}$ and travel health. ${ }^{2}$ Importation events in a country with no cases can lead to an exponential increase in case numbers within a short time period. ${ }^{3}$ As such, countries have implemented travel restrictions in response to the global rise; however, their effectiveness is debatable., ${ }^{4,5}$ Studies on the early introduction of the virus in a naive population can provide insight into the natural history of the disease and have implications for control measures.

Brunei Darussalam (pop. 459,500), a well-connected country vulnerable to multiple importation events, detected its first COVID-19 imported case on March 9, linked to an international super spreading event in Kuala Lumpur, Malaysia: the Sri Petaling Mosque Tablighi Jamaat cluster. This 4-day event (February 27-March 1, 2020) was attended by more than 16,000 members of the Tablighi, an apolitical Islamic group, from various parts of the world, including Brunei. ${ }^{6,7}$ Given the absence of widespread community transmission and a slowing in the trajectory of spread since the 100th confirmed case, Brunei's response to this first wave of the pandemic has been generally successful. ${ }^{8}$ To maintain this, a number of questions need to be addressed. First, what were the epidemiological characteristics of the cases observed so far? Second, what was the role of travel-related cases in driving the disease? Third, how infectious were the cases and how effective were the approaches to reducing transmission?

\footnotetext{
${ }^{*}$ Address correspondence to Liling Chaw, PAPRSB Institute of Health Sciences, Universiti Brunei Darussalam, Jalan Tungku Link, Gadong BE1410, Brunei Darussalam. E-mail: liling.chaw@ubd.edu.bn †These authors contributed equally to this work.
}

Here, we report findings from the first 135 COVID-19 cases, detected within the first 5 weeks of the local epidemic, along with their epidemiological, clinical, and transmission characteristics. As jurisdictions that have implemented lockdowns begin to bring the epidemic under control, ${ }^{9}$ our findings will be important to calibrate detection and response efforts in potential future waves of the pandemic.

\section{METHODS}

Case identification and contact tracing. The Brunei surveillance and contact tracing strategy has previously been described. ${ }^{7}$ Since January 23 , clinical and laboratory surveillance has been implemented across the country, and testing criteria have progressively expanded in scope (Supplemental Table 1). A confirmed case is someone who tested positive for SARS-CoV-2 through real-time reverse transcriptase-PCR (RT-PCR) test on nasopharyngeal (NP) swab. All laboratory-confirmed COVID-19 cases with symptom onset from March 9 to April 5, 2020 were included in this study and followed up until recovery or death.

Epidemiological investigation was conducted for each confirmed case, and information was collected on demographic characteristics, clinical symptoms, travel history, activity mapping 2 days before the onset of symptoms (or swab date for asymptomatic cases), and contact history. A close contact was any person living in the same household, or someone within $1 \mathrm{~m}$ of a confirmed case in an enclosed space for more than 15 minutes. All close contacts underwent RTPCR testing. Those who tested negative were quarantined at home for 14 days from last exposure, and those who later developed symptoms were retested. All confirmed COVID-19 cases were treated and isolated at the National Isolation Centre. Cases were discharged following two consecutive negative SARS-CoV-2 NP swabs collected at $\geq 24$-hour intervals. 
We categorized cases into two groups: imported cases (defined as individuals presumed to have acquired the infection outside Brunei) and locally transmitted cases (defined as those without a travel history).

Statistical analysis. An epidemic curve was constructed based on the date of symptom onset (for symptomatic and presymptomatic cases) or the date of NP swab collection (for asymptomatic cases). The duration between symptom onset to diagnosis date was calculated. The exposure period for imported cases and their close contacts was calculated as the duration between their return date to Brunei and their diagnosis/swab collection date. The incubation period was calculated as the duration between the known exposure date of confirmed cases and their symptom onset dates. The serial interval (SI) was calculated as the duration between the symptom onset dates for the primary case and that of a secondary case. Only symptomatic or presymptomatic infectorinfectee pairs with clear epidemiological links were included in the SI calculation. Group comparison was performed between the imported and local contact cases, using chi-square, Fisher's exact, or Mann-Whitney's tests as appropriate.

The estimation of the reproduction number (R0) (defined as the expected number of secondary cases infected by a primary case) was performed using two methods. The basic Ro was estimated from the mean SI and the exponential growth rate of the cumulative number of cases in the epidemic $\lambda(t)=\ln (Y[t]) / t$, using the formula $R 0=1+\operatorname{SI} \lambda+f(1-f)(\operatorname{SI} \lambda)^{2}$, where $f$ is the ratio of the infectious period to the SI. This assumes an exponential distribution, allowing for a range of reported values. ${ }^{10}$ The exponential growth phase between March 5 and 10 was chosen for the R0 estimation because it represents the initial growth of the epidemic curve (see Figure 1), and also because control measures were enhanced after March 9. Thus, this 6-day period represented more accurately the nature of SARS-CoV-2 transmission, in the absence of heightened measures. The epidemic growth rate and

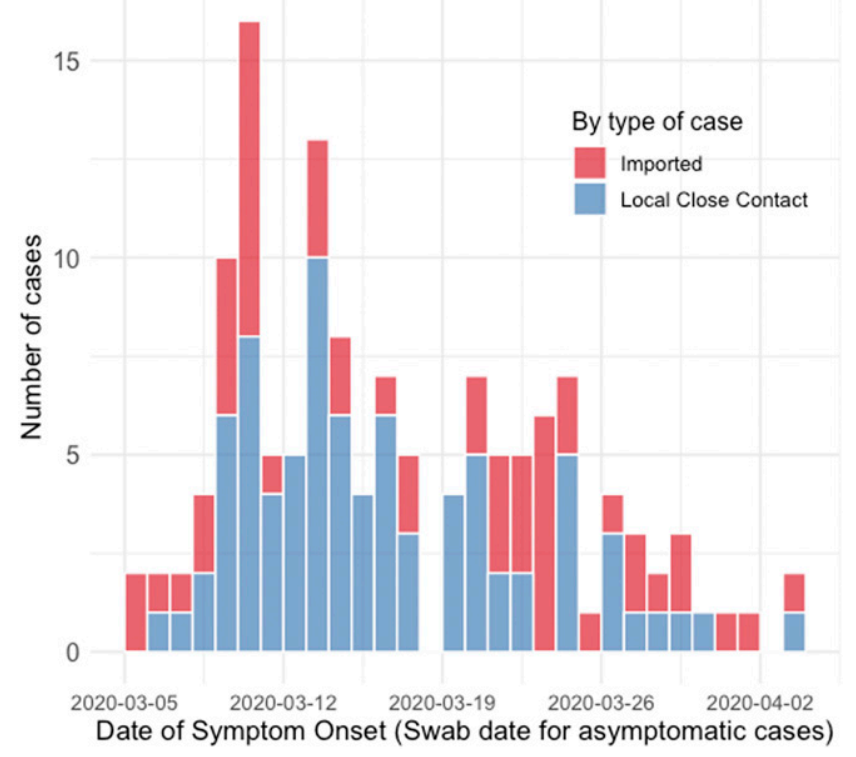

Figure 1. Epidemic curve for the first 135 COVID-19 cases in Brunei Darussalam, by imported (red bars) and locally transmitted cases (blue bars). This figure appears in color at www.ajtmh.org. doubling time were also calculated, based on established formulas. $^{11}$

We also estimated the time-varying reproduction number (Rt) to assess the effectiveness of epidemic control measures, based on methods proposed by Thompson et al. ${ }^{12}$ and using the EpiEstim 2.2 package. This Rt estimation uses case incidence data and SI distribution, and distinguishes between imported and locally transmitted cases. Following studies that use a nonnegative SI distribution to approximate the distribution of the generation time, ${ }^{13,14}$ we used a Gamma distribution and incorporated uncertainty in the parameters (SD of mean $=1, S D=0.5)$. The median $R t$ and $95 \%$ credible intervals for each day were estimated jointly from incidence data and from the posterior SI distribution, using a 6-day sliding window. This 6-day window reduces the bias associated with early estimations of Rt, as at least one average SI has passed. ${ }^{15}$

All analyses were conducted using Microsoft Excel (Microsoft Corp., Redmond, WA) and R (ver. 3.6.3, R Core Team, Vienna, Austria). ${ }^{16}$ A $P$-value $<0.05$ was considered as statistically significant. Ethics approval was granted by the University Research Ethics Committee, Universiti Brunei Darussalam (Ref: UBD/OAVCR/UREC/Apr2020-05).

\section{RESULTS}

Description of cases. A total of 135 cases were detected in the first 5 weeks, comprising 53 (39.3\%) imported and 82 $(60.7 \%)$ locally transmitted cases (Table 1). The median age was 36 years (ranging from 6 months to 72 years). Fifty-three $(39.3 \%)$ cases were female. Locally transmitted cases were significantly younger $(P=0.002)$ than imported cases and tend to be females $(P=0.008)$.

Eighty-one $(60 \%)$ cases developed symptoms, reported either during or before NP sample collection. Notably, we observed high proportions of presymptomatic and asymptomatic cases-41 (30.4\%) and 13 (9.6\%), respectively. In total, $61.5 \%$ of the cases were detected within 2 days of symptom onset or NP swab date. Among them, 32.6\% $(n=44)$ were detected on or before the day of symptom onset. No significant differences were observed between the imported and locally transmitted cases (Table 1). The most common reported symptoms were fever $(62.2 \%)$, sore throat $(62.2 \%)$, and cough (59.3\%) (Supplemental Table 2).

Among these 135 cases, three subsequently died from COVID-19 complications, giving a case fatality rate of $2.2 \%$. All three deaths were in men, aged 64,56 , and 67 years, respectively.

Impact of travel restrictions. Since late-January, travel restrictions were progressively implemented in response to the emerging regional and later global situation. Initially, travelers from Hubei Province, China, were restricted, whereas those from other parts of Mainland China underwent 14 days home quarantine. These restrictions were gradually tightened, first to travelers from Iran and Italy (the emerging epicenters) and then mandating quarantine for travelers from China and South Korea. These restrictions had a considerable impact on arrivals into Brunei. Immigration data indicate a $20.9 \%$ decrease in arrivals from January to March 2020 (872,315 people), compared with the same period in 2019 $(1,103,028$ people).

Outbound travel was restricted for all Brunei residents on March 15, and a ban on all foreign citizens entering the country was enacted on March 23. Beginning on March 20, all 
TABLE 1

Demographic and clinical characteristics of the first 135 COVID-19 cases in Brunei Darussalam

\begin{tabular}{|c|c|c|c|c|c|}
\hline & & All cases $(n=135), n(\%)$ & $\begin{array}{l}\text { Imported cases } \\
(n=53), n(\%)\end{array}$ & $\begin{array}{l}\text { Local contact cases } \\
(n=82), n(\%)\end{array}$ & $P$-value \\
\hline \multicolumn{2}{|l|}{ Median age (years) (IQR; min. to max.) } & $36.0(27 ; 0.5$ to 72$)$ & $39.0(27 ; 17$ to 68$)$ & $31.5(26 ; 0.5$ to 72$)$ & 0.002 \\
\hline \multirow[t]{7}{*}{ Age-group (years) } & $0-9$ & $7(5.2)$ & $0(0.0)$ & $7(8.5)$ & $<0.001$ \\
\hline & $10-19$ & $16(11.9)$ & $3(5.7)$ & $13(15.9)$ & \\
\hline & $20-29$ & 26 (19.3) & $7(13.2)$ & $19(23.2)$ & \\
\hline & 30-39 & $28(20.7)$ & $17(32.1)$ & $11(13.4)$ & \\
\hline & $40-49$ & $18(13.3)$ & $4(7.5)$ & $14(17.1)$ & \\
\hline & $50-59$ & $23(17.0)$ & $10(18.9)$ & $13(15.9)$ & \\
\hline & $60-69$ & 17 (12.6) & $12(22.6)$ & $5(6.1)$ & \\
\hline \multirow[t]{2}{*}{ Gender } & Female & $53(39.3)$ & $13(24.5)$ & $40(48.8)$ & 0.008 \\
\hline & Male & $82(60.7)$ & $40(75.5)$ & $42(51.2)$ & \\
\hline \multirow[t]{6}{*}{ Comorbidity } & Obesity & $6(4.4)$ & $3(5.7)$ & $3(3.7)$ & 0.679 \\
\hline & Heart disease & $6(4.4)$ & $3(5.7)$ & $3(3.7)$ & 0.679 \\
\hline & Respiratory disease & 7 (5.2) & $2(3.8)$ & $5(6.1)$ & 0.704 \\
\hline & Diabetes mellitus & 7 (5.2) & $5(9.4)$ & $2(2.4)$ & 0.111 \\
\hline & Hypertension & $18(13.3)$ & $11(20.8)$ & $7(8.5)$ & 0.067 \\
\hline & Hyperlipidemia & $18(13.3)$ & $12(22.6)$ & $6(7.3)$ & 0.018 \\
\hline \multirow[t]{3}{*}{ Symptom status* } & Symptomatic & $81(60.0)$ & $29(54.7)$ & 52 (63.4) & 0.596 \\
\hline & Presymptomatic & $41(30.4)$ & $18(34.0)$ & $23(28.0)$ & \\
\hline & Asymptomatic & $13(9.6)$ & $6(11.3)$ & $7(8.5)$ & \\
\hline \multirow{4}{*}{$\begin{array}{l}\text { Duration between symptom onset (or } \\
\text { swab taken) and diagnosis† (days) }\end{array}$} & -8 to 0 & 44 (32.6) & 17 (32.1) & 27 (32.9) & 0.665 \\
\hline & 1 to 2 & 39 (28.9) & $18(34.0)$ & $21(25.6)$ & \\
\hline & 3 to 5 & $26(19.3)$ & $8(15.1)$ & $18(22.0)$ & \\
\hline & $>5$ & 26 (19.3) & 10 (18.9) & 16 (19.5) & \\
\hline \multirow[t]{5}{*}{ Severity } & Asymptomatic & $13(9.6)$ & 7 (13.2) & $6(7.3)$ & 0.352 \\
\hline & Mild & 101 (74.8) & 36 (67.9) & 65 (79.3) & \\
\hline & Moderate & $14(10.4)$ & 7 (13.2) & 7 (8.5) & \\
\hline & Severe & $2(1.5)$ & $0(0.0)$ & $2(2.4)$ & \\
\hline & Critical & $5(3.7)$ & $3(5.7)$ & $2(2.4)$ & \\
\hline
\end{tabular}

$\mathrm{NP}=$ nasopharyngeal. The bold values indicate findings with $P$-value is $<0.05$.

${ }^{\star}$ Cases were classified as follows: 1) symptomatic, if symptoms were reported on or before NP swab collection day; 2) presymptomatic, if symptoms were reported after NP swab sample was taken but during admission; and 3) asymptomatic, if no symptoms were reported since NP swab collection day until the date of hospital discharge.

$\dagger$ This includes the asymptomatic cases, from whom the symptom onset date was replaced by the date of swab collection.

$¥$ Severity was classified as 1) asymptomatic, for those with no symptom throughout their disease; 2) mild, for patients who had uncomplicated upper respiratory tract infection symptoms and no radiological changes; 3) moderate, for patients with radiological changes but did not require supplemental oxygen; 4) severe, for patients who showed signs of severe pneumonia including tachypnea $>30$ /minute, $\mathrm{SpO}_{2}$ of $\leq 93 \%$ on room air, or abnormal arterial blood gases, as well as patients showing signs of sepsis with evidence of organ dysfunction; and 5 ) critical, for patients who developed septic shock, that is, persistent hypotension requiring vasopressors support to maintain mean arterial pressure $\geq 65$ and those who developed acute respiratory distress syndrome requiring ventilatory support.

individuals entering Brunei underwent RT-PCR testing on arrival and 14-day quarantine at a designated facility.

The origin country of imported cases changed over time. Cases were initially from Malaysia (20 cases), and, as the epidemic progressed globally, and overseas Brunei citizens were returning, imported cases were identified in travelers and returning residents from Indonesia $(n=14)$, United Kingdom $(n=$ 11), Thailand $(n=2)$, the United States $(n=2)$, Austria $(n=1)$, Cambodia $(n=1)$, Australia $(n=1)$, and the Philippines $(n=1)$.

Figure 1 shows the epidemic curve, by the date of symptom onset or NP swab date for asymptomatic cases. Detection of positive cases among local close contacts occurred very early in the epidemic. Coupled with the early detection of further generations (Supplemental Figure 1), this suggests a short time interval for transmission within the community.

Epidemic characteristics over time. The mean duration from symptom onset to diagnosis for local transmitted cases decreased from 9 days in the first week to -1.7 days in the fifth week of the epidemic (Figure 2A). Among imported cases, this reduction occurred between the third and fourth weeks of the epidemic (from 7.3 to 1.3 days, respectively), coinciding with implementation of quarantine and testing of all arrivals beginning March 20 (Figure 2A). This demonstrates the impact of increasingly stringent travel restrictions. There were no local infections linked to imported cases in the last 2 weeks of the epidemic.

Using the known return dates from imported cases $(n=53)$, the median duration in the exposure period to other contacts was 7 days (IQR $=5)$, ranging between 1 and 16 days. The reduction in the median exposure period between imported cases and their close contacts from their return to diagnosis was apparent between the third (8.5 days) and fourth weeks (4.0 days; Figure 2B). Summary statistics and distribution remained unchanged when the 15 local contact cases with known exposure dates were included. Among 82 local contact cases, 15 of them (18.3\%) had known dates of exposure to confirmed imported cases. Using these dates, the median incubation period was 5.0 days $(I Q R=5)$, ranging between 1 and 11 days.

Based on 59 symptomatic and presymptomatic infectorinfectee pairs, the mean SI was 5.4 days $(S D=4.5 ; 95 \% \mathrm{Cl}$ : 4.3, 6.5 [approximated using normal distribution]). The range for the SI was between -4 and 20 days (Figure 2C). Four pairs $(6.8 \%)$ had negative SI values. The median SI was relatively constant throughout the 4 weeks of the epidemic (Figure 2D).

Using the calculated mean SI and the 6-day growth phase of the epidemic, the growth rate was 0.54 /day, and the $\mathrm{R} 0$ in the early phase of the epidemic ranges between 3.9 and 6.0. The doubling time was 1.3 days.

Figure 3 shows the estimated Rt and the timing of the control measures implemented after March 11. The initial median reproduction number was estimated to be 2.2 (95\% credible intervals: $0.86,5.0)$ on the seventh day of the epidemic (March 11). The time-varying reproduction number gradually decreased after several control measures were put in place and was below one on the 13th day (March 17) and 

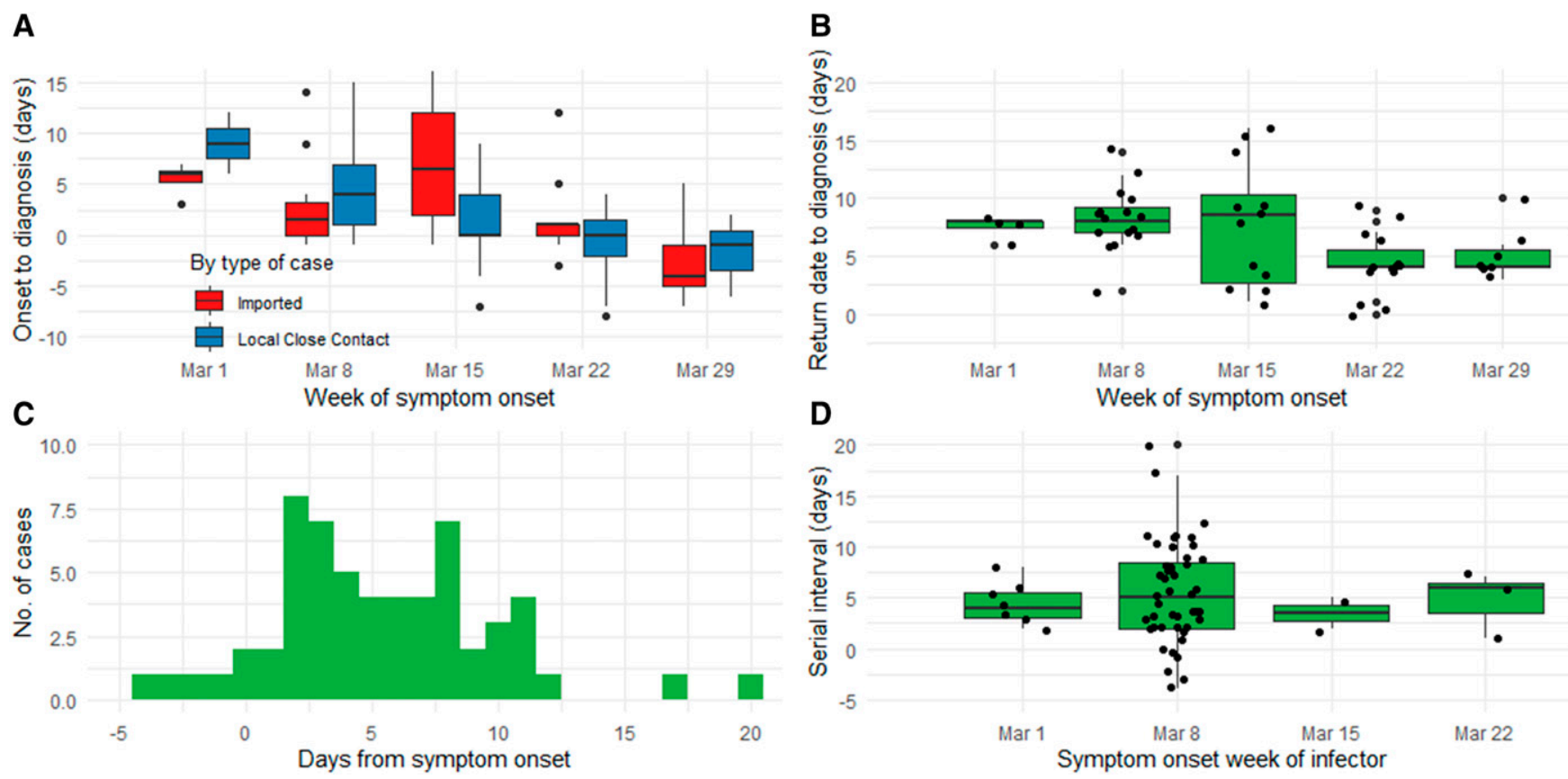

FiguRE 2. A, (Top left) Boxplot of the duration between symptom onset to diagnosis among symptomatic and presymptomatic cases only, by week of symptom onset and by type of case: imported (in blue) and local case contact (in red). Black dots above and below each boxplot indicate outliers; (B, top right) boxplot of the exposure period of the 53 imported cases to their local contact cases (using their return dates to Brunei as the start point), by week of symptom onset. Black dots represent each case, jittered for visual clarity; (C, bottom left) histogram showing the serial interval (SI) distribution for the 59 symptomatic infector-infectee pairs; (D, bottom right) boxplot showing variations in the SI distribution by the symptom onset week of the infector. Black dots represent each case, jittered for visual clarity. This figure appears in color at www.ajtmh.org.

reached 0.20 (95\% credible intervals: $0.06,0.49)$ on the 30 th day (April 3). However, because these control measures were implemented quickly over a short time period, it is difficult to attribute the observed reduction in $\mathrm{Rt}$ to a specific intervention.

\section{DISCUSSION}

Key findings. A total of 135 cases (53 imported and 82 locally transmitted) were reported from the start of the epidemic on March 5 until the first week of April. All locally

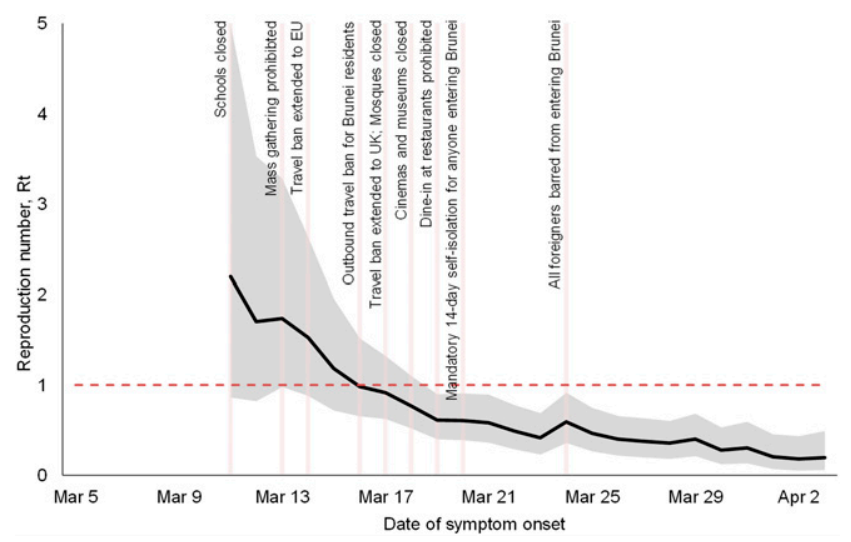

FIGURE 3. Time-varying reproduction number (Rt) estimates of the COVID-19 epidemic in Brunei. A 6-day sliding window was used. The black solid line is the estimated median Rt, and the gray areas are the $95 \%$ credible intervals. This figure appears in color at www.ajtmh.org. transmitted cases could be traced to an importation event, and there were no cases detected without a clear epidemiological link. Brunei managed to successfully control the first wave of the COVID-19 pandemic. The R0 was between 3.9 and 6.0, and the epidemic had a doubling time of 1.3 days during the exponential phase. There was a rapid decline such that by day 13 , the Rt was under one.

Estimating the reproduction number. Assuming the ratio of the infectious period to the SI $(f)$ is 0.3 , the R0 was 5.6, higher than those estimated by the WHO (1.9-2.5), but within those estimated in other studies from China ${ }^{17-19}$ and Europe. ${ }^{20}$ Our observations of negative SIs pose challenges for specifying $f$. Hence, we reported $\mathrm{R} 0$ as a range covering all plausible values of $f$, using the mean $S I$ value from our data and with assumption of exponential growth. Then, Rt was estimated from the 7 th to the 30 th day. The $95 \%$ credible intervals of the initial $R t$ value (0.86-5.0) are consistent with those of the Ro estimate.

Test, isolate, and trace. We observed that the approach of isolation of confirmed cases, as well as tracing and quarantine of their contacts, was associated with a significant reduction in the effective reproduction number. By the time the early physical distancing measures had been implemented, the Rt had reduced from 5.6 (ranging between 3.9 and 6.0) at the start to $2.2(95 \%$ credible bands: $0.86,5.0)$ by the seventh day (March 11). Our findings on the importance of the test, isolate, and trace approach are consistent with observational studies from Hong Kong ${ }^{21}$ and Singapore. ${ }^{22}$

We highlight three characteristics of the approach implemented in Brunei. First, testing was conducted on a significant scale. Even before detection of the first case, testing was 
already conducted for all inpatient pneumonia cases, in addition to those who met the suspect case definition; 24-hour testing centers were established within 2 days of the detection of the first case (March 11), and all contacts were tested regardless of symptom status. ${ }^{8}$ The relatively high proportion of asymptomatic $(9.6 \%)$ and presymptomatic (30.4\%) cases identified demonstrates both the breadth of testing and the speed at which cases were identified.

Second, all confirmed cases (regardless of disease severity and symptom status) were isolated in a dedicated isolation facility and remained isolated until two negative results were obtained from RT-PCR specimens at 24-hour intervals. This reduced the chance of household transmission, which could not be excluded if cases were allowed to isolate at home. ${ }^{23}$ Finally, contact tracing was conducted for each case, using a variety of tools including case interview, workplace assessment, and mobile phone data. Contacts were placed on 14-day home quarantine, and inperson spot checks with penalties for noncompliance were also conducted.

Travel restrictions and other non-pharmaceutical interventions. We report several characteristics of SARSCoV-2 that make effective isolation and contact tracing challenging, including high transmissibility, a relatively short SI (mean $\mathrm{SI}=5.39$ days), and a high proportion of asymptomatic and presymptomatic cases suggesting the potential for silent transmission. As such, the test, isolate, and trace approach was supplemented with physical distancing measures to increase the likelihood of achieving sustained control. ${ }^{24}$

Restricting travel is one measure by which countries have responded to the COVID-19 pandemic. ${ }^{25}$ By the time Brunei implemented an exit travel ban and restricted the entry of foreign citizens in the country, the Rt was already decreasing and had neared one. Nonetheless, we suggest that reducing ongoing vulnerabilities to importation events, through restrictions on incoming travelers, and requirements for testing and quarantine for all arrivals in the country limited additional spread, consistent with a reduction in mean time from symptom onset to diagnosis observed for imported cases following implementation of mandatory quarantine and testing for all arrivals. Modeling studies suggest a role for travel restrictions in containing the epidemic, with one model estimating that travel restrictions in Wuhan reduced case importations elsewhere by nearly $80 \%$ through mid-February. ${ }^{26}$

Although various other physical distancing measures were implemented in Brunei, including school closures; prohibition on mass gatherings, cinemas, and religious services; and dine-in restrictions, importantly, no lockdown was implemented, and there were no generalized stay-at-home orders. Most businesses and government agencies were able to operate. These suggest that effective test, isolate, and trace approaches were able to control the epidemic with moderate levels of physical distancing. ${ }^{27}$ This finding corroborates the experience of other countries. In Hong Kong, case isolation and contact tracing were combined with other physical distancing measures (but no lockdown), which resulted in an estimated effective reproduction number near 1 for 8 weeks. ${ }^{28}$ In South Korea, testing and tracing have been combined with school closures and remote working. ${ }^{29}$

Even with the best efforts at testing, case identification, and quarantine, the potential for widespread community transmission of COVID-19 is clear. Once the disease is established, suppression may require the implementation of severely disruptive social distancing measures. ${ }^{30,31}$

Limitations. Our study had several limitations. First, although we can be reasonably confident of having identified most cases since March, given more restrictive testing criteria in January and February, we were unable to account for potential importation events that may have occurred before detection of the first case. Second, the generalizability of our results are limited because of lack of community transmission, small number of cases, and a lack of cases in settings such as residential care facilities and dormitories. Third, due to the potential for presymptomatic infection of SARS-CoV-2, using the SI distribution to approximate the generation time distribution is problematic. We had not accounted for negative SIs with the use of the Gamma distribution, and thus may have overestimated Rt to fit incidence data. One way to account for negative $\mathrm{SI}$ is to use a deconvolution approach using the incubation period distribution to recover the generation time distribution. ${ }^{32}$ However, this assumes that the generation time and incubation period distributions are independent, which may not be appropriate. Finally, given the limited data available and the analytic methods used, we could not directly estimate the effectiveness of other non-pharmaceutical interventions, such as face mask wearing, personal hygiene practices, and voluntary reductions in mobility.

\section{CONCLUSION}

Swift control of COVID-19 in Brunei was achieved through a combination of public health measures, focusing on a testisolate-trace approach supplemented by travel restrictions and general physical distancing measures, but no actual lockdown. As of August 5, 2020, Brunei has recorded a total of 141 confirmed COVID-19 cases, with the last case reported on May 6. On May 18, Brunei commenced its de-escalation plan in a stepwise approach including reopening of mosques, schools, and other indoor facilities in phases, while still maintaining its border control and enhancing the surveillance program (including testing of high-risk groups such as those working in residential institutions, healthcare workers, and also individuals at relatively high risk of developing severe complications). In conjunction with easing of physical distancing restrictions, a mobile application was rolled out on May 14 to speed up the process of contact tracing. Its public uptake among the general adult population was $>90 \%$. Along with timely reimposition of physical distancing measures, if necessary, these can help maintain suppression of a second wave in Brunei.

Received June 30, 2020. Accepted for publication August 6, 2020.

Published online August 14, 2020.

Note: Supplemental figure and tables appear at www.ajtmh.org.

Acknowledgment: Publication charges for this article were waived due to the ongoing pandemic of COVID-19.

Authors' addresses: Justin Wong and Mohammad Fathi Alikhan, Disease Control Division, Ministry of Health, Gadong, Brunei Darussalam, E-mails: justin.wong@moh.gov.bn and fathi.alikhan@moh. gov.bn. Liling Chaw and Lin Naing, PAPRSB Institute of Health Sciences, Universiti Brunei Darussalam, Gadong, Brunei Darussalam, E-mails: liling.chaw@ubd.edu.bn and ayub.sadiq@ubd.edu.bn. Wee Chian Koh, Centre for Strategic and Policy Studies, Gadong, Brunei Darussalam, E-mail: wckoh@csps.org.bn. Sirajul Adli Jamaludin, 
Environmental Health Division, Ministry of Health, Gadong, Brunei Darussalam, E-mail: sirajul.jamaludin@moh.gov.bn. Wan Wen Patricia Poh, Department of Dental Services, Ministry of Health, Gadong, Brunei Darussalam, E-mail: patricia.poh@moh.gov.bn.

This is an open-access article distributed under the terms of the Creative Commons Attribution (CC-BY) License, which permits unrestricted use, distribution, and reproduction in any medium, provided the original author and source are credited.

\section{REFERENCES}

1. Heymann DL, Shindo N, 2020. COVID-19: what is next for public health? Lancet 395: 542-545.

2. Wilder-Smith A, Chiew CJ, Lee VJ, 2020. Can we contain the COVID-19 outbreak with the same measures as for SARS? Lancet Infect Dis 20: e102-e107.

3. Leung K, Wu JT, Liu D, Leung GM, 2020. First-wave COVID-19 transmissibility and severity in China outside Hubei after control measures, and second-wave scenario planning: a modelling impact assessment. Lancet 395: 1382-1393.

4. Kraemer MUG et al., 2020. The effect of human mobility and control measures on the COVID-19 epidemic in China. Science 368: 493-497.

5. Iken O, Abakporo U, Ayobami O, Attoye T, 2020. COVID-19: travel health and the implications for sub-Saharan Africa. Travel Med Infect Dis 35: 101645.

6. Mat NFC, Edinur HA, Razab MKAA, Safuan S, 2020. A single mass gathering resulted in massive transmission of COVID-19 infections in Malaysia with further international spread. $J$ Travel Med 27: taaa059.

7. Chaw L, Koh WC, Jamaludin SA, Naing L, Alikhan MF, Wong J, 2020. SARS-CoV-2 transmission in different settings: analysis of cases and close contacts from the Tablighi cluster in Brunei Darussalam. medRxiv, doi: 10.1101/2020.05.04.20090043.

8. Wong J, Koh W, Alikhan M, Abdul Aziz A, Naing L, 2020. Responding to COVID-19 in Brunei Darussalam: lessons for small countries. J Global Health 10: 010363.

9. Lavezzo E et al., 2020. Suppression of COVID-19 outbreak in the municipality of Vo, Italy. medRxiv, doi: 10.1101/2020.04.17.20053157.

10. Lipsitch $M$ et al., 2003. Transmission dynamics and control of severe acute respiratory syndrome. Science 300: 1966-1970.

11. Vynnycky E, White RG, 2010. An Introduction to Infectious Disease Modelling. Oxford, United Kingdom: Oxford University Press.

12. Thompson RN et al., 2019. Improved inference of time-varying reproduction numbers during infectious disease outbreaks. Epidemics 29: 100356.

13. You C, Deng Y, Hu W, Sun J, Lin Q, Zhou F, Pang CH, Zhang Y, Chen Z, Zhou X-H, 2020. Estimation of the time-varying reproduction number of COVID-19 outbreak in China. Int $\mathrm{J} \mathrm{Hyg}$ Environ Health 228: 113555.

14. Zhang $\mathrm{J}$ et al., 2020. Evolving epidemiology and transmission dynamics of coronavirus disease 2019 outside Hubei province, China: a descriptive and modelling study. Lancet Infect Dis 20: 793-802.

15. Cori A, Ferguson NM, Fraser C, Cauchemez S, 2013. A new framework and software to estimate time-varying reproduction numbers during epidemics. Am J Epidemiol 178: 1505-1512.
16. R Core Team, 2020. R: A Language and Environment for Statistical Computing. Vienna, Austria: R Foundation for Statistical Computing.

17. Tang B, Wang X, Li Q, Bragazzi NL, Tang S, Xiao Y, Wu J, 2020. Estimation of the transmission risk of the 2019-nCoV and its implication for public health interventions. J Clin Med 9: 462.

18. Zhou W, Wang A, Xia F, Xiao Y, Tang S, 2020. Effects of media reporting on mitigating spread of COVID-19 in the early phase of the outbreak. Math Biosci Eng 17: 2693-2707.

19. Shen M, Peng Z, Xiao Y, Zhang L, 2020. Modelling the epidemic trend of the 2019 novel coronavirus outbreak in China. bioRxiv, doi: 10.1101/2020.01.23.916726.

20. Yuan J, Li M, Lv G, Lu ZK, 2020. Monitoring transmissibility and mortality of COVID-19 in Europe. Int $J$ Infect Dis 95: 311-315.

21. Wu $P$ et al., 2020. Suppressing COVID-19 transmission in Hong Kong: an observational study of the first four months. Res Square (Preprint version 1).

22. $\mathrm{Ng} \mathrm{Y}$ et al., 2020. Evaluation of the effectiveness of surveillance and containment measures for the first 100 patients with COVID-19 in Singapore - January 2-February 29, 2020. MMWR Morb Mortal Wkly Rep 69: 307-311.

23. Hellewell $\mathrm{J}$ et al., 2020. Feasibility of controlling COVID-19 outbreaks by isolation of cases and contacts. Lancet Global Health 8: e488-e496.

24. Kucharski AJ et al., 2020. Early dynamics of transmission and control of COVID-19: a mathematical modelling study. Lancet Infect Dis 20: 553-558.

25. Daon Y, Thompson RN, Obolski U, 2020. Estimating COVID-19 outbreak risk through air travel. $J$ Travel Med: taaa093. Available at: https://doi.org/10.1093/jtm/taaa093.

26. Chinazzi $M$ et al., 2020. The effect of travel restrictions on the spread of the 2019 novel coronavirus (COVID-19) outbreak. Science 368: 395-400.

27. Wilasang C, Sararat C, Jitsuk NC, Yolai N, Thammawijaya P, Auewarakul P, Modchang C, 2020. Reduction in effective reproduction number of COVID-19 is higher in countries employing active case detection with prompt isolation. J Travel Med: taaa095. Available at: https://doi.org/10.1093/jtm/ taaa095.

28. Cowling BJ et al., 2020. Impact assessment of non-pharmaceutical interventions against coronavirus disease 2019 and influenza in Hong Kong: an observational study. Lancet Public Health 5: e279-e288.

29. Government of the Republic of Korea, 2020. Flattening the Curve on COVID-19-How Korea Responded to a Pandemic Using ICT. Seoul, Republic of Korea: Government of the Republic of Korea.

30. Sjödin H, Wilder-Smith A, Osman S, Farooq Z, Rocklöv J, 2020. Only strict quarantine measures can curb the coronavirus disease (COVID-19) outbreak in Italy, 2020. Eurosurveillance 25: 2000280.

31. Lau H, Khosrawipour V, Kocbach P, Mikolajczyk A, Schubert J, Bania J, Khosrawipour T, 2020. The positive impact of lockdown in Wuhan on containing the COVID-19 outbreak in China. J Travel Med 27: taaa037.

32. Knight J, Mishra S, 2020. Estimating effective reproduction number using generation time versus serial interval, with application to COVID-19 in the Greater Toronto Area, Canada. medRxiv, doi: 10.1101/2020.05.24.20109215. 